\title{
Independent Authentication Protocol in Tactical Network Environment Using Hash Lock Approach
}

\author{
Jin-suk Kang
}

\begin{abstract}
Ubiquitous computing in being actively researched and one of the main technology in ubiquitous computing environments is recognized as RFID system. The RFID system has much benefits but simultaneously has some problems such as user's privacy violation. In this paper, in order to improve the survivability of its nodes, it should build available simulation surrounding sensor nodes. Also, In the proposed cryptosystems we use a new hash function for user authentication and a stream cipher based on LFSR(Linear Feedback Shift Register) for message encryption and decryption. Moreover, each algorithm is programmed with $\mathrm{C}$ language and simulated on IBM-PC system and we analyze the randomness properties of the proposed algorithms by using statistical tests.
\end{abstract}

Index Terms-Tactical network environment, hash lock approach, hash function, sensor network.

\section{INTRODUCTION}

RFID, its application, standardisation, and innovation are constantly changing. Its adoption is still relatively new and hence there are many features of the technology that are not well understood by the general populace. Developments in RFID technology continue to yield larger memory capacities, wider reading ranges, and faster processing. It's highly unlikely that the technology will ultimately replace bar code even with the inevitable reduction in raw materials coupled with economies of scale, the integrated circuit in an RF tag will never be as cost-effective as a bar code label. However, RFID will continue to grow in its established niches where bar code or other optical technologies aren't effective. If some standards commonality is achieved, whereby RFID equipment from different manufacturers can be used interchangeably, the market will very likely grow exponentially [1], [2].

A more complex proposal is the "Hash Lock" approach counteracting unauthorized reads: A tag does not reveal its information unless the reader has sent the right key being the preimage to the hash value sent by the tag. The scheme requires implementing cryptographic hash functions on the tag and managing keys on the backend. This is regarded as economic for the near future. Unfortunately, the scheme offers data privacy but no location privacy since the tag can be uniquely identified by its hash value. Another drawback is that the key is sent in plain text over the forward channel which can be eavesdropped easily from a large distance. The extended scheme called "Randomized Hash Lock" ensures

Manuscript received April 9, 2015; revised June 26, 2015.

Jin-suk Kang is with Jangwee Research Institute for National Defence, Ajou University, San 5, Woncheon-dong, Yeongtong-gu, Suwon 443-749, Republic of Korea (e-mail: jskang01@ajou.ac.kr). location privacy but is not scalable for a huge number of tags since many hash-operations must be performed at the back-end and it additionally relies on the implementation of a random number generator in the tags to randomize tag responses. Such devices need sources for physical randomness so that the implementation is rather complex and expensive [3], [4].

\section{THE HASH-LOCK APPROACH}

The Hash-Lock approach proposed by Weis et al. [5]. uses the concept of locking and unlocking the tag to allow access. The security of the Hash-Lock approach uses the principle based on the difficulty of inverting a one-way hash function. The scheme makes use of a back-end database to provide correct reader to tag identification and the concept of meta-ID stored in each tag. To lock the tag the reader sends a hash of a random key, as the meta-ID, to the tag. i.e. meta-ID<-hash(key). The reader then stores the meta-ID and key in the back end database. While locked, the tag only responds with the meta-ID when queried. As shown in Fig. 1, to unlock the tag, the reader will query the tag for the meta-ID. The reader will then use the meta-ID to lookup a key and ID for the tag in the database. If the meta-ID is found, the reader then sends the key to the tag in an attempt to unlock the tag. The tag hashes the key and compares the results against the meta-ID stored in the tag.

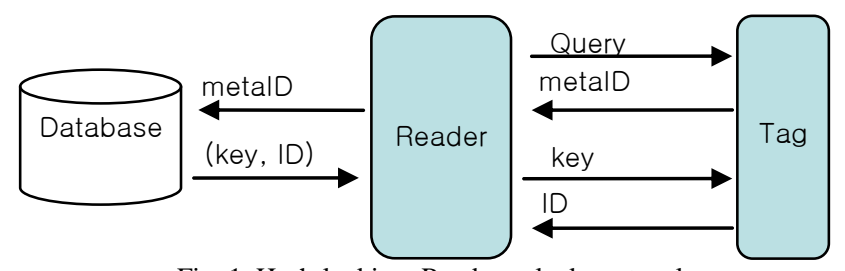

Fig. 1. Hash-locking: Reader unlock protocol.

\section{A. Hash Function Algorithm}

The Cryptographic hash functions are playing very important roles in modern cryptology such as checking the integrity of information or increasing efficiency of authentication code and digital signature. While compared with general hash functions used in non cryptographic computer applications, although both cases are functions from domain to range, they're different from each other in several important aspects. Also, the hash function outputs the value called has value or has code of fixed length by the input of messages having random length. In more strict words, the hash function h corresponds text alignment of random length as $\mathrm{n}$ bit text alignment having fixed length.

When domain is called $D$ and range is called $R$, the function 
d $h: D \rightarrow R(|D|>R) \quad$ is a many-to-one corresponding function. Accordingly, the collision exists for the has function in general. For example, assuming function $h$ as the one having input value of $t$ bit and output value of $n$ bit, the number of input values while $\mathrm{h}$ has randomness corresponds to each output value. Accordingly, two input values selected at random with probability $2^{-n}$ gets to have same output value regardless of the $t$ value.

The handling process of most has functions is the repetitive one hashing the input of random length by divided processing of successive fixed blocks. First, the input $X$ becomes padded to become a multiple of block length and divided from $X_{1}$ to $t$ number of blocks as $X_{t}$. The hash function $\mathrm{h}$ is described as follows.

$$
\begin{aligned}
& H_{0}=I V \\
& H_{i}=f\left(H_{i-1}, X_{i}\right), \\
& 1 \leq i \leq t, \\
& h(X)=H_{t}
\end{aligned}
$$

Here, $f$ is the compress function), $H_{i}$ is the chaining variable between $i-1$ and $i$, while $I V$ is the initial value. The general structure of repetitive has function using compressed function is like the Fig. 2.

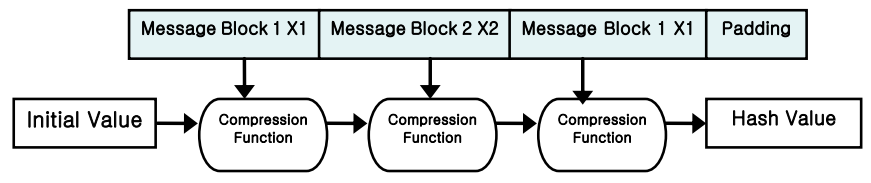

Fig. 2. Structure of the hash-function with recurrent.

The calculation of hash value is dependent on the chain variable. While starting the hash calculation, this chain variable gets to have the fixed initial value expressed as the part of algorithm. The compressed function renews this chain variable by getting the message block as input until it becomes hashed. The processes get repeated in cycles for all message blocks and the last value gets output as hash value on the same message [2]. The hash function gets classified into 3 types depending on which structure is used as internal compressed function.

1) Hash-Functions based Block Cipher

2) Hash-Functions based Modular Calculation

3) The other Hash-Functions

The exclusive hash function has fast processing speed and they're the functions specially designed for hashing regardless of other system sub factors. The exclusive has function proposed until now has the structure based on MD4 [6] designed by Rivest in 1990. There are MD5 [3], SHA-1 [7], RIPEMD-160 [8] and HAVAL [9] for hash functions of MD series being widely used at this time.

When a specific hash function is assigned, although it is ideal to verify the lowest limit on complications attacking the hash function for the establishment of safe hash functions, such method isn't known for the most part in reality and the applicable known complication of the attack becomes considered as the security of hash function for the most part. If hash value is assumed as uniform probability variable. The following are well-known facts.

- For the $n$ bit hash function $h$, the guessing attack to discover preimage and second preimage with $2^{n}$ operation.

- For the attacker that is able to select messages, the birthday attack is able to discover the collision message pair $M, M^{\prime}$ with about $2^{n / 2}$ operation.

If $n$ bit hash function satisfies the following two characteristics, it gets to have an ideal security. Once the hash value is given, the discovery of preimage and second preimage require $2^{n}$ operation.

\section{B. Suggestion of Algorithm}

The exclusive hash function proposed in this thesis has been designed at 32 bit process using addition, subtraction, multiplication and exclusive logical sum operations that are basic operations of the CPU. Although Boolean function has been used in order to raise nonlinearity in case of MD series exclusive hash functions, the $x^{-1}$ operation was used in this thesis. Although the operation of getting inverse elements generally takes long time, the operation was performed in advance to form a reference table because it is an inverse element at $G F\left(2^{2}\right)$. All operations are accomplished as $32 \mathrm{bit}$ module and six 32 bit registers a, b, c, d, e and $\mathrm{f}$ get the final hash value as chain variables. These registers become initialized as $h_{0}$ value and its value is as follows.

$$
\begin{aligned}
& a=0 x 01234567 ; b=0 x \mathrm{efcdab} 89 ; c=0 x 98 \mathrm{badcef} \\
& d=0 x 10325476 ; e=0 x \mathrm{c} 3 \mathrm{~d} 2 \mathrm{e} 1 \mathrm{f} 0 ; f=0 x 5 \mathrm{a} 3 \mathrm{cf} 01 \mathrm{~d}
\end{aligned}
$$

The 256 bit message blocks are divided into 32 bit module and $h_{i}$ is renewed as $h_{i+1}$ due to the operation being used and connected as initial value of $\{x 0, x 1, x 2 \ldots \ldots x 7\}$ registers, key scheduling is performed in between and the current value $h_{i+1}$ of registers $a, b, c, d, e$ and $f$ are finally made at the feedforward stage. The expression method of $C$ programming language has been used for the formulae to express algorithm.

\section{1) The Structure of Algorithm}

save_abcdef

$\operatorname{pass}(a, b, c, d, e, f, 3)$

key_schedule

$\operatorname{pass}(a, b, c, d, e, f, 5)$

key_schedule

$\operatorname{pass}(a, b, c, d, e, f, 7)$

feedforward

a) Feedforward is save_abcdef: save initial value $h_{t}$

$$
a a=a ; b b=b ; c c=c ; d d=d ; e e=e ; f f=f ;
$$

b) Construct a pass $(a, b, c, d, e, f$, mul) is,

round ( $a, b, c, d, e, f, x 0, \mathrm{mul})$;

round ( $b, c, d, e, f, a, x 1, \mathrm{mul})$;

round ( $c, d, e, f, a, b, x 2, \mathrm{mul})$;

round ( $d, e, f, a, b, c, x 3, \mathrm{mul})$;

round ( $e, f, a, b, c, d, x 4$, mul);

round $(f, a, b, c, d, e, x 5, \mathrm{mul})$; 
round ( $a, b, c, d, e, f, x 6$, mul); round $(f, b, d, a, c, e, x 7, \mathrm{mul})$;

Here, construct a round ( $\mathrm{a}, \mathrm{b}, \mathrm{c}, \mathrm{d}, \mathrm{e}, \mathrm{f}, \mathrm{X}, \mathrm{mul})$ is,

$$
\begin{aligned}
& f^{\wedge}=\mathrm{X} ; a \text {-=Gen_32 }(f, f, f, f) ; \\
& f^{\wedge}=a ; b+=\text { Gen_32 }(f, f, f, f) \text {; } \\
& b *=\text { mul; } \\
& f^{\wedge}=b ; c+=\text { Gen_32 }(f, f, f, f) \text {; } \\
& c^{*}=\text { mul; } \\
& f^{\wedge}=c ; d+=\text { Gen_32 }(f, f, f, f) \text {; } \\
& d^{*}=\text { mul; } \\
& f^{\wedge}=d ; e+=\text { Gen_32 }(f, f, f, f) \text {; } \\
& e^{*}=\text { mul; }
\end{aligned}
$$

Here, the Gen_32( ) function is the one which gets four 32 bit registers as input to use first, second, third and fourth 8 bit as the input of S-box and makes 32 bit value with corresponding S-box output.

c) Key_schedule is,

$$
\begin{gathered}
x 0-=x 7^{\wedge} 0 x \text { A5A5A5A5; } x 1^{\wedge}=x 0 ; x 2+=x 1 ; \\
x 3-=x 2 ; \wedge((\sim x 1)<<7) ; x 4^{\wedge}=x 3 ; x 5+=x 4 ; \\
x 6=x 5^{\wedge}((\sim x 4)>>23) ; x 7^{\wedge}=x 6 ; x 0+=x 7 ; \\
x 1-=x 0^{\wedge}((\sim x 7)<<7) ; x 2^{\wedge}=x 1 ; x 3+=x 2 ; \\
x 4-=x 3^{\wedge}((\sim x 2)>>23) ; x 5^{\wedge}=x 4 ; x 6+=x 5 ; \\
x 7^{\wedge}=x 6^{\wedge} 0 x 01234567 ;
\end{gathered}
$$

It is like the following. Here the $>>,<<$ are left and right logical shift operators.

d) Feedforward is,

$$
\begin{aligned}
& a^{\wedge}=a a ; b-=b b ; c+=c c ; \\
& d d^{\wedge}=d d ; e-=e e ; f+=f f ;
\end{aligned}
$$

Here the $a, b, c, d, e$ and $f$ registers are $h_{i+1}$ which is the halfway hash value of $192 \mathrm{bit}$ and becomes the final hash value after termination of algorithm. Accordingly, the $32 \mathrm{bit}$

\begin{tabular}{|c|c|c|c|c|c|c|c|c|c|c|c|c|c|c|c|}
\hline $0 \mathrm{xa} 5$ & oxa4 & $0 \times 33$ & $0 \mathrm{x} 41$ & 0xee & $0 \mathrm{xf} 9$ & $0 \mathrm{xd} 7$ & $0 \times 60$ & $0 \times 16$ & $0 \mathrm{xe} 6$ & $0 \mathrm{x} 8 \mathrm{~b}$ & $0 \times 58$ & $0 \times 9 c$ & 0x99 & $0 \times 51$ & $0 \times 91$ \\
\hline $0 x 6 a$ & $0 \times 52$ & $0 \times 12$ & $0 \times 97$ & $0 \mathrm{xb} 2$ & $0 \mathrm{x} 4 \mathrm{c}$ & $0 \mathrm{x} 4 \mathrm{~d}$ & $0 \mathrm{xb} 1$ & $0 \times 2 f$ & $0 \times 83$ & $0 \mathrm{xbb}$ & $0 \times 7 f$ & $0 \mathrm{xdf}$ & $0 \times 15$ & $0 \mathrm{xbf}$ & $0 \times 7 e$ \\
\hline $0 \times 54$ & $0 \mathrm{xec}$ & $0 \times 48$ & $0 \times 68$ & $0 \times 68$ & 0x1f & $0 \mathrm{xbc}$ & $0 \times 2 e$ & $0 \times 38$ & $0 \times 5 b$ & $0 \times 47$ & $0 \times 5 c$ & $0 \mathrm{xd} 1$ & $0 \mathrm{x} 08$ & 0xaf & $0 \times 59$ \\
\hline $0 \mathrm{xe} 0$ & $0 \times 44$ & $0 x b 6$ & $0 \times 13$ & 0xaa & $0 \times 50$ & $0 \mathrm{xc} 8$ & 0x04 & $0 \times 98$ & 0xa9 & $0 x f d$ & $0 \times 20$ & $0 \mathrm{xa} 8$ & $0 \mathrm{x} 9 \mathrm{~d}$ & $0 \times 5 e$ & $0 \times 19$ \\
\hline $0 \mathrm{x} 4 \mathrm{~b}$ & $0 \times 2 a$ & $0 \times 17$ & Oxac & $0 \mathrm{x} 45$ & 0x95 & $0 \times 06$ & $0 \times 56$ & $0 \times 55$ & $0 \times 84$ & $0 x f 8$ & 0xal & $0 \times 3 f$ & $0 \times 3 d$ & $0 \times 76$ & $0 \mathrm{x} 0 \mathrm{e}$ \\
\hline $0 \times 7 d$ & $0 \times 6 c$ & $0 \mathrm{xda}$ & $0 \mathrm{xfa}$ & $0 \mathrm{xd} 4$ & $0 \times 2 c$ & $0 \mathrm{x} 4 \mathrm{f}$ & $0 \mathrm{xd} 8$ & $0 x 9 f$ & $0 \times 21$ & $0 \times 65$ & $0 \mathrm{xc} 3$ & $0 \mathrm{xa} 0$ & 0xef & $0 \mathrm{xdb}$ & $0 \mathrm{xf} 6$ \\
\hline 0xl1 & $0 x 6 f$ & $0 \times 43$ & $0 \times 79$ & $0 \times 3 a$ & $0 \times 67$ & $0 \mathrm{xfe}$ & $0 \times 64$ & $0 \times 34$ & $0 x d c$ & $0 \times 49$ & $0 \times 86$ & $0 \mathrm{x} 05$ & 0x93 & $0 \times 63$ & $0 \times 28$ \\
\hline $0 \times 2 d$ & $0 \mathrm{xf1}$ & $0 x a 3$ & $0 x 61$ & $0 \times 89$ & 0x09 & $0 \times 71$ & $0 \times 25$ & $0 \times 35$ & $0 \mathrm{xcc}$ & $0 \mathrm{xb} 9$ & $0 \times 14$ & $0 \mathrm{x} 4 \mathrm{e}$ & $0 \mathrm{xf} 2$ & $0 \mathrm{xfb}$ & $0 \times \mathrm{xf} 7$ \\
\hline $0 x d 2$ & $0 \times 70$ & $0 \times 74$ & $0 \times 7 a$ & $0 \mathrm{xfc}$ & $0 \mathrm{x} 9 \mathrm{e}$ & $0 \times 37$ & $0 \times 73$ & $0 \mathrm{xd} 5$ & $0 \times f 0$ & $0 x b d$ & $0 \times 82$ & $0 \times 62$ & $0 \mathrm{xca}$ & $0 \times 4 a$ & $0 \mathrm{xe} 4$ \\
\hline $0 x d d$ & $0 \mathrm{xcd}$ & $0 \times 23$ & $0 \times 72$ & $0 \mathrm{x} 1 \mathrm{~d}$ & $0 \times 02$ & $0 \mathrm{xa} 7$ & $0 \times 40$ & 0 xe8 & $0 \times 3 e$ & 0xe9 & $0 \times 3 c$ & $0 \times 5 a$ & $0 \mathrm{x} 8 \mathrm{~d}$ & $0 \times 66$ & $0 \mathrm{xc} 1$ \\
\hline $0 \mathrm{xc} 9$ & $0 \times 92$ & $0 \times 57$ & $0 \mathrm{xe} 3$ & $0 \mathrm{x} 0 \mathrm{c}$ & $0 \mathrm{x} 0 \mathrm{a}$ & $0 \times 1 c$ & $0 \times 30$ & $0 \mathrm{x} 0 \mathrm{~b}$ & $0 \mathrm{x} 01$ & $0 \times 77$ & $0 \mathrm{xea}$ & $0 \mathrm{xd} 0$ & $0 x 88$ & $0 \mathrm{x} 0 \mathrm{~d}$ & $0 \times 00$ \\
\hline $0 \mathrm{xb} 8$ & $0 x d e$ & $0 \mathrm{xe} 7$ & 0xad & $0 \mathrm{xc} 5$ & $0 \times 6 e$ & $0 \times 96$ & $0 \mathrm{xb} 7$ & $0 \times 31$ & $0 \mathrm{x} 03$ & $0 \times 80$ & $0 \times 69$ & $0 \times 9 a$ & $0 \times 5 f$ & $0 \times 1 a$ & $0 \times 1 b$ \\
\hline $0 \mathrm{xff}$ & $0 \mathrm{xc} 2$ & $0 \mathrm{xc} 0$ & $0 \times 3 b$ & $0 x d 6$ & $0 \times a 2$ & $0 \mathrm{xcb}$ & $0 \times 29$ & $0 \times 7 c$ & $0 \mathrm{xd} 4$ & $0 \mathrm{xc} 4$ & $0 \times 10$ & $0 \times 1 e$ & $0 \times 81$ & $0 \times 53$ & $0 \mathrm{xb} 5$ \\
\hline $0 \times 7 b$ & $0 \times 27$ & $0 \mathrm{x} 0 \mathrm{f}$ & $0 \mathrm{xeb}$ & $0 \mathrm{xd} 3$ & $0 \times 24$ & $0 \times 22$ & $0 \times 36$ & $0 \mathrm{xf5}$ & $0 \mathrm{x} 6 \mathrm{~d}$ & 0xbe & $0 \mathrm{xba}$ & $0 \mathrm{xc} 6$ & $0 \times 42$ & $0 \times 75$ & $0 \times 26$ \\
\hline 0xe1 & $0 \times 94$ & $0 \mathrm{x} 8 \mathrm{f}$ & $0 \times 5 d$ & $0 x a 6$ & $0 \times 32$ & $0 \mathrm{xc} 7$ & $0 \times 78$ & $0 x b 3$ & $0 \mathrm{xb} 0$ & $0 \times f 3$ & $0 x d 9$ & $0 \mathrm{xcf}$ & $0 \times 87$ & $0 \mathrm{xe} 5$ & $0 \times 2 b$ \\
\hline Oxed & $0 \times 85$ & $0 \times 07$ & $0 x e 2$ & $0 \mathrm{xab}$ & $0 \times 90$ & $0 \times 6 b$ & $0 \mathrm{xb} 4$ & $0 \times 46$ & $0 \mathrm{x} 8 \mathrm{e}$ & $0 \times 18$ & $0 \times 9 b$ & $0 x 8 a$ & Oxae & $0 \mathrm{x} 8 \mathrm{c}$ & $0 \times 39$ \\
\hline
\end{tabular}
SRES(Signed Response) and the encryption key 64bit $K_{c}$ are finally generated by the following formula.

$$
\begin{gathered}
\text { SRES }=a^{\wedge} b^{\wedge} c^{\wedge} d, \\
K_{C}=e f,
\end{gathered}
$$

\section{2) S-box}

S-box has used the $x^{-1}$ operation in order to raise nonlinearity and the operation of getting inverse elements generally require a lot of operation time. But the operation was performed in advance to form a reference table because it is an inverse element at $G F\left(2^{8}\right)$ Because inverse element of 0 doesn't exist, the value of 0 is corresponded. But because this isn't cryptologically safe, the exclusive-OR has been performed for $0 x a 5$ value to form a table having 256 eight bit values. The S-box table is shown on Table I.

TABLE I: S-BOX TABLE

\section{DESIGN OF INDEPENDENT AUTHENTICATION PROTOCOL}

\section{A. Experimental}

\section{1) Simulation environment}

In this paper, in order to improve the survivability of its nodes, it should build available simulation surrounding under the surrounding sensor nodes (it mean 4 component; survivability of sensor nodes - available battery, the output of sensor nodes - available area for search, the communication path of sensor nodes - generation of routing table, bandwidth of sensor nodes - the size of data transfer). Visual simulation environment configuration with Fig. 3.

\section{2) Tiny OS}

As in Fig. 4 is Tiny Operating System such as existent UNIX in 32bits computer-on-a-chip a number Megabyte memory need. Sensor node has memory of $10 \mathrm{Kbyte}$ degrees of 8-16bit computer-on-a-chip in sensor network. There are TinyOS, MicroC/OS, Nucleus, Nano-X to available Operating System. TinyOS embedded hardware directly and need one physical address space as one Process. Memory is suitable Operating System to sensor network because memory allocates compile dynamically and use Function Call instead of software signaling or exception processing [9], [10]. 


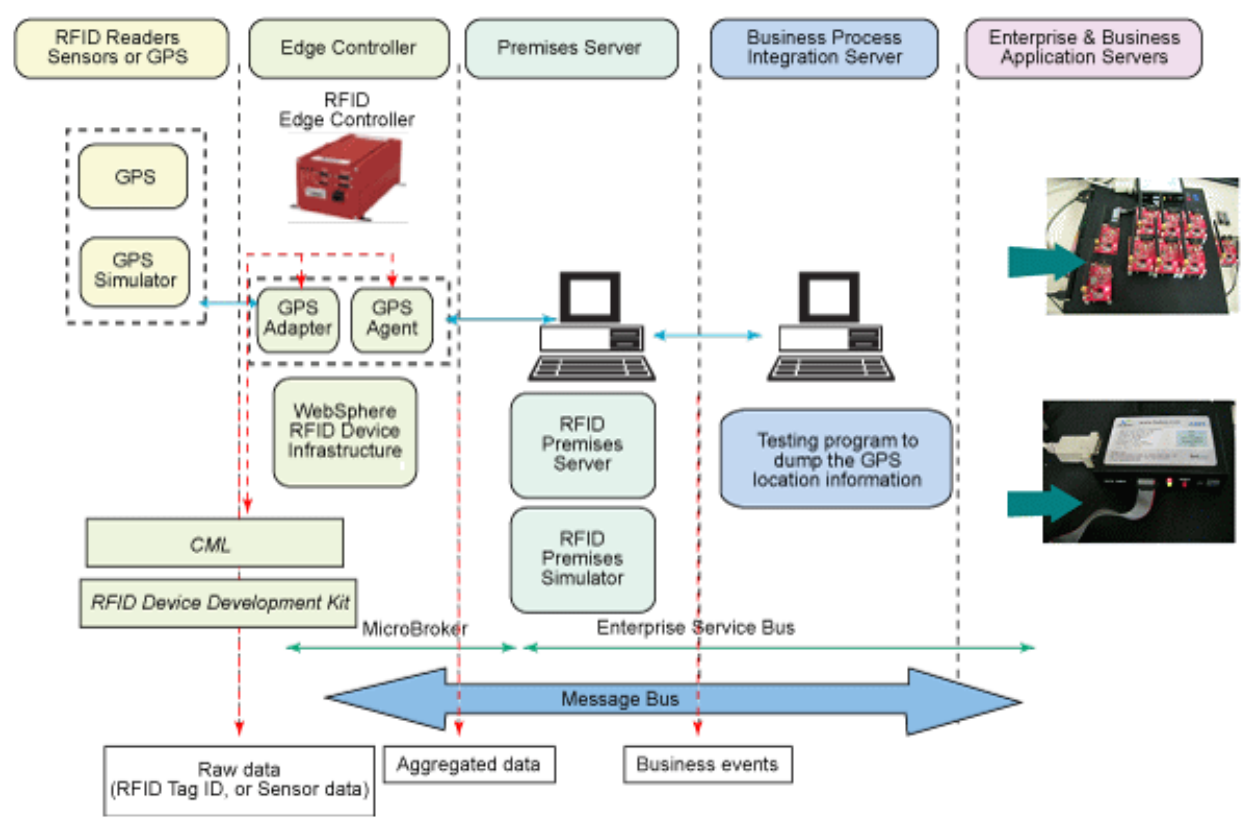

Fig. 3. Simulation environment architecture.

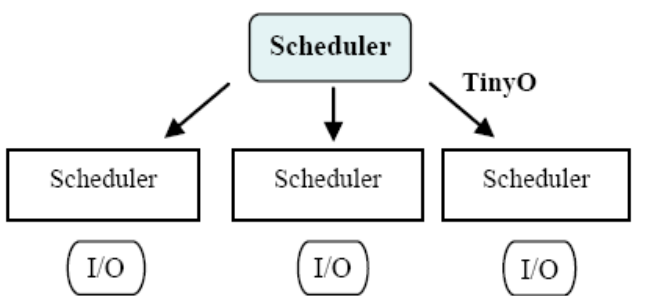

Fig. 4. TinyOS architecture.

\section{B. Design of Sense Node Data Modules}

\section{1) Design of message manager}

Message manager serial communication data be delivered through UIC(User Interface Command) main form and compose this in a few arrangement form(Array List). Message manager has following function.

- Log Service: Data original save

- Topology service: Paint topology on screen

- Node data history: Data history per node show

\section{2) Module function}

UART is delivered to high position application receiving data of sensor node. Must establish UART for this and serial communication and need connection attribute value setting for this. Data delivered through Database serial communication input database log leave.

\section{CONCLUSION}

The work by Sarma et al. [10] predicts that over the next several years, development of low-cost tags in the range of US $\$ 0.05$ or less will continue to present a challenge to manufacturers. Low-cost tags will remain extremely resource scarce, passively powered, and have limited memory resources comprised of several hundred bytes, as opposed to kilobytes. The range of communications will be a few meters, with a limit on computational power. Using standard cryptographic security mechanisms will exceed the capability of these devices. To meet these challenges, more work must be done to develop new hardware-efficient hash functions within low-cost RFID tags, along with new lightweight cryptographic primitives and protocols. Any new and efficient functions need to take into account the limited resources of low-cost RFID tags.

In this paper the threats to personal privacy and security that exist in low-cost RFID tags have been identified, goals and assumptions defined, and proposed solutions to address these privacy and security risks analyzed. Based on the comparison of these solutions, the selective blocker tag provides the best solution satisfying most requirements.

As RFID technology advances allowing "smarter" tags, the line between RFID devices, smart cards, and general-purpose computers will blur. Today's research benefiting RFID devices will aid in the development of secure ubiquitous computing systems in the future.

\section{ACKNOWLEDGMENT}

This research was supported by basic science research program through the NRF funded by MEST (No.2010-0020985 and No. 2014R1A2A1A11049469) and also supported by the Technology Commercialization support program, Ministry of Agriculture, Food and Rural Affairs (No.113038-03-1-HD020).

\section{REFERENCES}

[1] V. Dixit, H. K. Verma, and A. K. Singh, "Comparision of various Security Protocols in RFID," International Journal of Computer Applications, vol. 24, no. 7, June 2011.

[2] L. Yang, P. Yu, W. Bailing, Q. Yun et al., "Hash-based RFID mutual authentication protocol," International Journal of Security and Its Applications, vol. 7, no. 3, May 2013.

[3] M. O. Balitanas and T. Kim, "Review: Security threats for RFID-sensor network anti-collision protocol," International Journal of Smart Home, vol. 4, no. 1, pp. 23-36, January 2010.

[4] G. Avoine and P. Oechslin, "A scalable and provably secure hash-based RFID protocol," in Proc. IEEE International Conference on Pervasive Computing and Communications, 2005, pp. 110-114.

[5] S. A. Weis, "Security and privacy in radio-frequency identification devices," MIT Master of Science Thesis, submitted May 2003.

[6] MIT Auto-ID Center. [Online]. Available: http://www.autoidcenter.org 
[7] S. Sarma, D. Brock, and D. Engels, "Radio frequency identification and the electronic product code," Micro IEEE Trans, vol. 21, no. 6, pp. 50-54, 2001.

[8] D. McCullough. (January 13, 2003). RFID tags: Big Brother in small packages. CNet, Available: http://news.com.com/2010-1069-980325.html

[9] A. Juels and R. Pappu, "Squealing Euros: Privacy Protection in RFID-Enabled Banknotes," in Financial Cryptography, R. Wright, Ed., 2003, vol. 2742, pp. 103-121.

[10] S. Sarma, S. Weis, and D. Engels, "Radio-frequency identifiers: Security Risks and Challenges," CryptoBytes, vol. 6, no. 1, 2003.

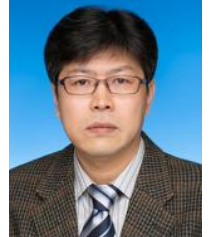

Jin-suk Kang received his B.S. degree in information engineering from Cheju National University, Jeju, Korea, in 1999; got his M.S. and Ph.D. degrees in computer engineering from Cheju National University, Jeju, Korea, in 2001 and 2005, respectively. From 2006 to 2009 , he was with the University of Incheon, Korea, as a research professor. From February 2009 to March 2010, he worked with Chungbuk National University, Korea, as a visiting professor. Since March 2010, he has been with the Jangwee Research Institute for National Defence, Ajou University, Suwon, Korea, where he is currently a research professor. His research interests include the areas of multimedia, computer vision, human-computer interaction, mobile computing and embedded system, etc. 